\title{
Memory Cache
}

National Cancer Institute

\section{Source}

National Cancer Institute. Memory Cache. NCI Thesaurus. Code C63626.

RAM memory that is set aside as a specialized buffer storage that is continually updated; used to optimize data transfers between system elements with different characteristics. 